DE DE GRUYTER

OPEN

DOI 10.2478/pesd-2014-0004

PESD, VOL. 8, no. 1, 2014

\title{
THE LAND'S SUSCEPTIBILITY, DUE TO ATMOSPHERIC PRECIPITATIONS, WITHIN THE CATCHMENT AREA OF CÂLNĂU
}

\author{
Dana Maria (Oprea) Constantin ${ }^{1}$, Emil Cîrciumaru ${ }^{2}$, Victor Viorel \\ Vătămanu ${ }^{3}$
}

Key words: precipitation, impact, Angot parameter, modelling processes, Câlnău River

Abstract. The climatic factors, generally, and the precipitation amounts recorded, especially, constitute some of the factors which condition the development and intensity of actual geomorphologic processes. One of the most employed climatic parameters for determining the land's susceptibility to atmospheric precipitations, with real and concrete applications into the dynamic geomorphology, is the Angot factor.

The precipitations represent a crucial meteorological element in initiating, sustaining and reactivating some geomorphologic processes, acting as a modelling agent for every type of landscape. This influence can be highlighted by quantifying the values of some characteristic variables (length, frequency and intensity), as well as temporally confining individual and successive sequences, which present a certain degree of susceptibility.

The general objective of this research, through the analysis of precipitations and the Angot parameter, is to identify, on one hand, the months, seasons or years with a very high or very low susceptibility to the occurrence of bank erosion phenomena, and on the other, to examine the value variations of this parameter across the year. The purpose is to recognize a cumulative of pluviometric conditions which could contribute to inducing, in general, the modelling processes.

The study of land's susceptibility to atmospheric precipitations was conducted within the morpho-hydrographic drainage system of Câlnău.

1 Assistant Dr., University of Bucharest, Faculty of Geography, Department of Meteorology-Hydrology, danamartines@yahoo.com

2 Prof. dr. National College "Iulia Hașdeu" from Bucharest, sorinemilghe@yahoo.com

3 agro-meteorologist - dr. engineer, Regional Meteorology Centre Oltenia - Craiova, victor.vatamanu@gmail.com 


\section{Introduction}

The atmospheric precipitations are being produced in different quantities and discontinuously throughout time, having as a principal characteristic the dissimilarity between space and time. The general circulation of the atmosphere, the thermodynamic convection and the particularities of the underlying surface specific to the physical-geographic space of the morpho-hydrografic system Câlnău are all factors which influence the occurrence of precipitations.

The most common used parameter in determining the topographic surface's susceptibility to erosion from atmospheric precipitations is the Angot factor. This coefficient is used in the dynamic geomorphology of drainage basins.

Since the theme of this article represents the analysis of the relationship between precipitations and land degradation, henceforth we will depict the atmospheric precipitations through monthly and annual registered means, as maximum fallen quantities in 24 hours and as the determined monthly pluviometric parameter Angot, applied in the study of annual variations of atmospheric precipitations.

The data base utilized for this particular precipitation research and for establishing the Angot monthly pluviometric parameter, is comprised from meteorological observations carried out by the weather station Buzău (alt. 97 m, $45^{\circ} 09^{\prime}$ lat. $\mathrm{N}, 25^{\circ} 5^{\prime}$ long E.) for the time frame $1980-2012$.

The evaluation of atmospheric precipitations, but most importantly the role played, as impact factor in the land's subsidence, is performed for Câlnău's catchment basin.

\section{Geographic features of Câlnău's catchment area}

The drainage basin of Câlnău is located in the south-eastern part of Romania and the arch of Outer-Carpathians, composing some of Buzău's catchment area, being the last major sub-basin of this system, before joining waters with Siret, as it can be seen in Figure 1.

The actual geographic landscape of Câlnău's basin reflects modifications along the lines of north-south and west-est, generally as a result of its geographic position. Therefore in the nordic and central parts it can be distinguished a hilly scenery, whilst the south is characterised by a mountain glacier environment.

Câlnău River originates from Costomirului Hills, at an altitude of $730 \mathrm{~m}$, whereas the confluence with Buzău River occurs at 92 de m, near Buzău municipality, within its alluvial cone.

The landscape actively influences the intensity of current developing processes through its fragmentation grade, Câlnău's basin, being affected by landslides and the presence of torrential valleys. The predominant hypsometric 
level reads between $350-550 \mathrm{~m}$, characterized by powerful fragmentation, due to an intermittent hydrologic chain, with a degree of one and two.
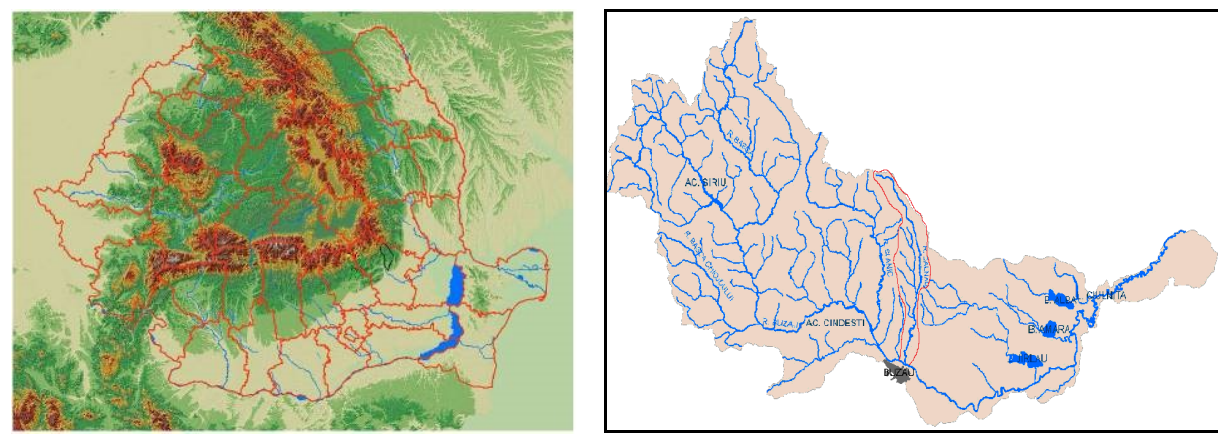

Fig. 1 The location of Câlnău's sub-basin within Romania and Buzau's watershed

From the biogeography standpoint, within this watershed occurs the transition among forest and forest-steppe ecoregions, allowing the expansion of deciduous forest region. The vegetation diminishes the impact of precipitations on soil, retaining a portion of fallen rainfall and adjusting the watercourse on slopes and in riverbeds, consequently attenuating soil erosion.

The soil, in the central and southern parts, is categorized as levigated chernozems, whilst in the north the soils are classified as alluvial-clay and pseudorendzina levigated. In the formation process of artificial discharge these soils constitute a major influence, acting as an interface between precipitations and discharge.

Presently, the anthropogenic pressure is not manifested through the rising number of population, but through neglecting requirements of sustainable development, as a result of unawareness or indifference.

\section{Morphometric features of Câlnău's basin}

Câlnău's watershed (Figure 2) is catalogued as a small drainage basin, as it is regarded a unit of the largest catchment area in the country, Siret's basin. With a surface of $209 \mathrm{~km}^{2}$, it represents $3.8 \%$ of the total drainage area of Buză and $0.08 \%$ of the total area of Romania, and its drainage system is viewed as a fusiform, elongated shape, Câlnău River has a length of $62 \mathrm{Km}$ and a medium debit of $0.45 \mathrm{~m} / \mathrm{s}$. On Câlnău's channel there are no body of water accumulations, however along its length have been built 30 dams, in the time spam $1972-1982$, which allowed for a while the retention of small masses of water, influencing positively both liquid and solid discharges. 


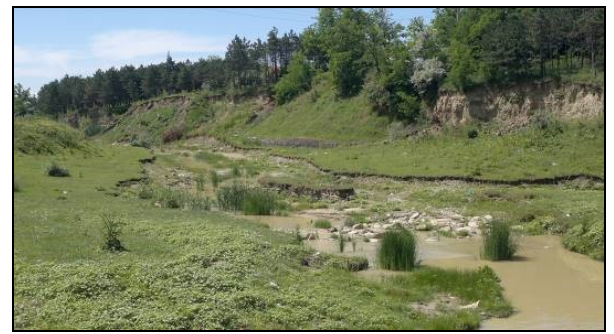

Fig. 2 - Câlnău's River valley. Source: personal archive

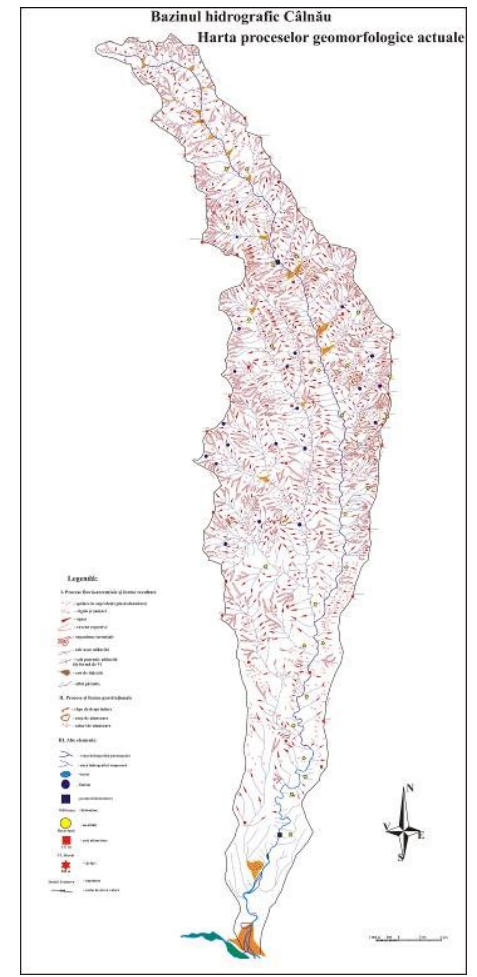

Fig. 3 - Map of actual geomorphologic processes in the drainage area of CâlnăuSource: adaptation of a topographic map 1: 50000

Câlnău River holds the record, nationally, for the level of water turbidity (at PH Potâtnichești - $72500 \mathrm{mg} / \mathrm{l}$ ), an aspect which is reflected in the origin of the word "Câlnău", translating to "muddy water". After the arrangements to combat 
erosion have begun to yield results, the level of turbidity started recording a significant decline.

The general inclination of the thalweg is approximately $17 \%$, and after it enters the area of the sub-Carpathian glacis the slope becomes $\sim 4 \%$ stretching to the section of the meander course, and under $2 \%$ downwards from that sector.

The surface waters grid consists of semi permanent rivers, temporary rivulets, lakes and wetlands reduced in size. The system features 16 important affluent streams, of which 11 are on the left, and the rest on right, therefore attributing an asymmetric layout (Figure 3), specific to subsequent valleys, with arrangements and confluence angles characteristic to every sector.

The hydrographical network of Câlnău receives an input both from surface waters (rainfall and snow), which represent the predominant means of supplementation and amounts to $60 \%$ of the annual volume $(55-65 \%$ rainfall and $35-45 \%$ snow), and from subterranean sources. The discharge regime can be characterized through a period of high flood, during spring, and series of outrushes, throughout the year.

\section{Atmospheric precipitations in the catchment area of Câlnău}

Atmospheric precipitations have a major component, from the regime point of view and the space - time repartition, great variability and discontinuity in time and space.

The precipitation regime is the result of interactions amongst general genetic factors and local factors.

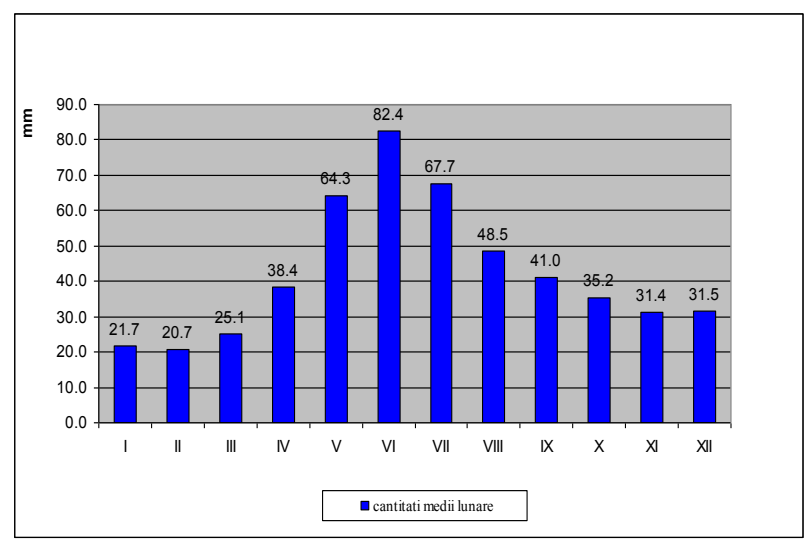

Fig. 4 - Mean values (mm) of monthly multiannual precipitations, at Buzău weather station, during 1980 - 2012. Data ANM processed 
From the analysis of multiannual monthly precipitation values (1980 - 2012), supplied by Buzău's weather station, overall it is evidently a rise in values from February, with a recorded value of $20.7 \mathrm{~mm}$, until June $(82.4 \mathrm{~mm})$, after which towards the end of the year and in January $(21.7 \mathrm{~mm})$, these gradually decline, as it can be observed in Figure 4.

The pluviometric minimum, recorded in February, is due to the higher frequency of continental air mass from winter months, while the pluviometric maxima is the consequence of local processes intensifying thermal and dynamic convection which generates cumulonimbus clouds. Amid these extreme values, the range of mean monthly multiannual data also registers between 25 and $65 \mathrm{~mm}$, from March to May, and between 68 and $31 \mathrm{~mm}$, from July to December.

In general the average multiannual quantity, for Buzău meteorological data, in the time period $1980-2012$, is of $507.8 \mathrm{~mm}$.

The annual behaviour of solar radiation, determines in its interaction with the active-underlying surface periodic developments of atmospheric circulation, which in its turn adjusts the method, amount, duration and frequency of precipitations across the seasons. Researching the amount of mean precipitations from the whole year it can be acknowledged a variation of data from month to month and from a season to another imposed by the general atmospheric circulation and the intensity of thermal convection.

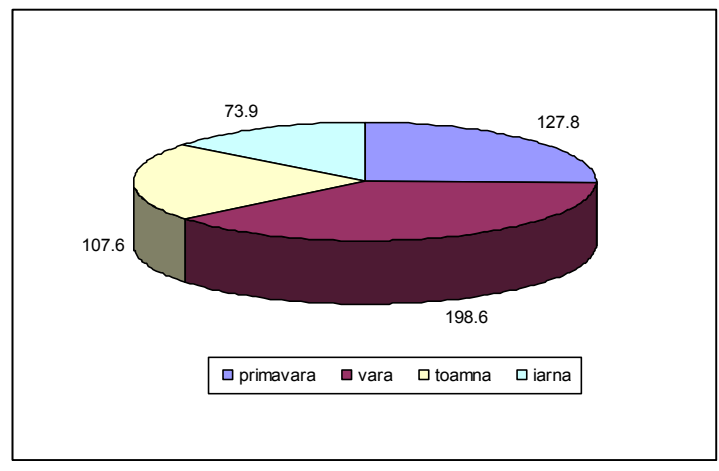

Fig. 5 - Mean values (mm) of multiannual season precipitations, at Buzău weather station, during 1980 - 2012. Data ANM processed

According to season data it is observed that the maximum value is recorded in the summer, of $198.6 \mathrm{~mm}$, while the minimum value occurs in the winter, of 73.9 $\mathrm{mm}$. For the transition seasons, registered data within the time period $1980-2012$, at Buzău weather station, are: $127.8 \mathrm{~mm}$ - spring and $107.6 \mathrm{~mm}$ - fall. We recognize that during summer, medium amounts of rainfall are recorded, being 2.6 
times bigger that those during winter while the difference between the sums of spring and fall is of $20.2 \mathrm{~mm}$, in the favour of spring rain events, as depicted in Figure 5.

The maximum amount of precipitations fallen in 24 hour are either determined by a powerful local convection, or by the passing of a cold front. It accounts as a great vulnerability which affects the water volume registered in 24 hours, as it can sometime exceed the medium quantity from the respective month, detailed in Figure 6.

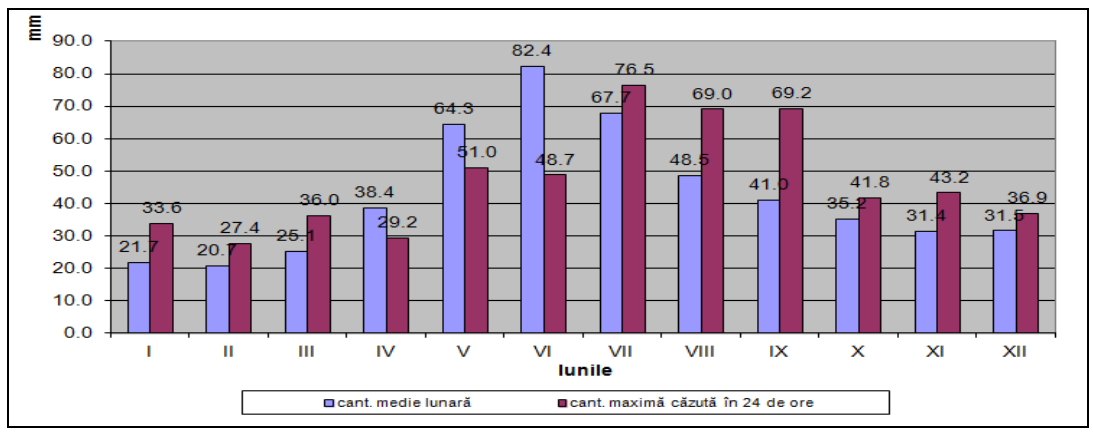

Fig. 6 - Mean values (mm) of monthly multiannual and fallen amounts in 24 hours of rainfall, at Buzău weather station, during 1980 - 2012.Data ANM processed

The maximum volume of precipitations fallen in 24 hours registers the biggest values during summertime, of $76.5 \mathrm{~mm}$ in July and $69.0 \mathrm{~mm}$ in August, whilst in wintertime the maximum diurnal amount of water are generally small due to the reduced content of water vapour, resulted from cold air masses, measuring 36.9 $\mathrm{mm}$ in December and $33.6 \mathrm{~mm}$ in January.

Values of maximum volume of precipitation surpassing the mean monthly quantities are detected both in the months of September and May, counting 69.2 $\mathrm{mm}$ and $51.0 \mathrm{~mm}$, respectively. Only in the following months: April, May and June, the values of maximum precipitation quantities dropped in 24 hours are lower than the mean monthly multiannual.

In the yearly warm period, these recorded volumes of rainfall in 24 hours present a downpour aspect and can be categorized as a climatic hazard.

Another parameter employed in the methodical interpolation of data regarding atmospheric precipitations, is represented by the variability of monthly and annual precipitation amounts. Consequently, it can be highlighted the infrequent variations of precipitations from year to year. In general, the largest amounts of precipitation fall during years when cyclonic activity is very intense and persistent, and at a monthly level, the biggest quantities of recorded rainfall arise in the summer 
months. The reduced volume of precipitations occurs during the years and months with frequent and persistent anticyclone conditions, characterized through a considerable prevail of polar continental air masses, with a deficiency in water vapour. Predominantly, droughty years or with scarce precipitations are found clustered, in groups of $2-3$ years.

In Figure 7, it can be asserted that the largest annual precipitations were recorded in the years: 1980 with $680.7 \mathrm{~mm}, 1984$ with $618.6 \mathrm{~mm}, 1991$ with 717.3 mm, 1997 with $729.3 \mathrm{~mm}, 1999$ with $671.4 \mathrm{~mm}, 2005$ with $753.7 \mathrm{~mm}$ and 2012 with $598.9 \mathrm{~mm}$. Moreover, it can be observed that the highest value was measured in 2005 , exceeding by $48.2 \%$ the mean multiannual quantity.

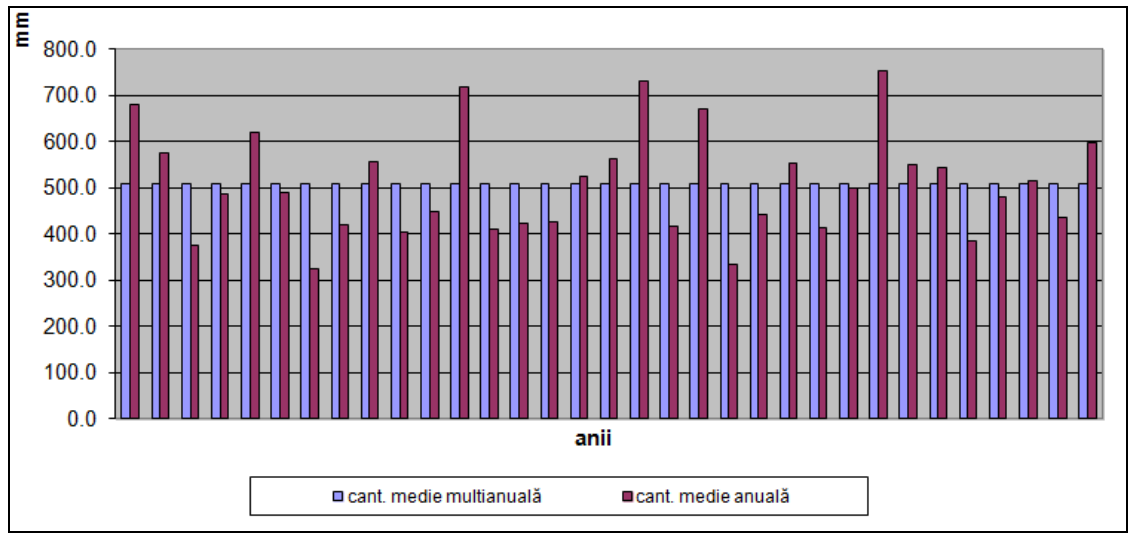

Fig. 7 - Variability of annual precipitation amounts at Buzău weather station, during the period 1980 - 2012. Data ANM processed

Similarly, the lowest amounts of annual precipitations were produced in the following years: 1982 with $375.2 \mathrm{~mm}, 1986$ with $323.7 \mathrm{~mm}$, which is also the minimum value for the investigated period, smaller by $36.3 \%$ than the mean multiannual quantity, 1989 with $403.3 \mathrm{~mm}, 2000$ with $333.3 \mathrm{~mm}$ and 2008 with $386.2 \mathrm{~mm}$. In these precipitation scarce years, were even noted the presence of the most months with pluviometric deficiency.

Throughout the seasons of winter, spring and summer for the periods of 1992 - 1994, $2000-2001$ and $2008-2009$, across the whole country, and implicitly for the catchment area of Câlnău, were registered a deficit of $20-30 \%$ than the normal. Within the $1980-2012$ time frame was counted a number of 18 negative deflections and 15 positive deviations. The greatest negative fluctuation is documented in 1986 of $184.1 \mathrm{~mm}$, followed by the one from 2000 of $174.5 \mathrm{~mm}$, beside the mean multiannual volume of $507.8 \mathrm{~mm}$; on the other hand the record 
positive anomaly is produced in 2005 of $245.9 \mathrm{~mm}$, pursued by the value from 1997 of $221.5 \mathrm{~mm}$.

Furthermore, great fluctuations are represented even at the level of monthly mean precipitation amounts, as it appears in Figure 8.

The greatest amounts of monthly precipitations occur especially in the summer and least during winter, the maximum value being recorded in May 2012 as $213.0 \mathrm{~mm}$, with $148.7 \mathrm{~mm}$ more than multiannual monthly average. The smallest value among the biggest volumes of monthly rainfalls is the one registered in January 1998 de $50.2 \mathrm{~mm}$, with additional $28.5 \mathrm{~mm}$ than the multiannual monthly mean.

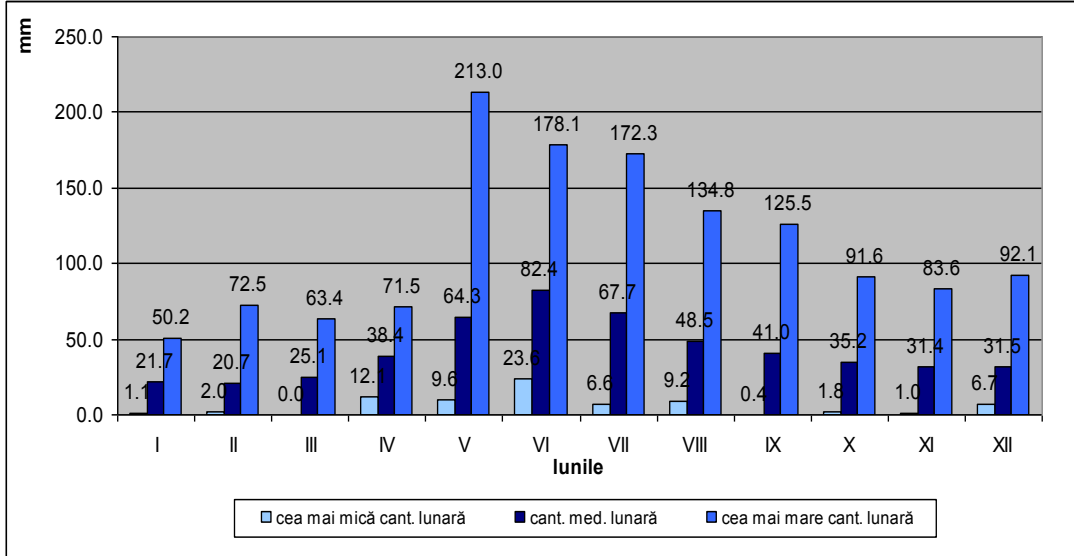

Fig. 8 - Variability of monthly precipitation amounts, at Buzău weather station, during the period 1980 - 2012. Data ANM processed

Contrary, the minimum amounts of monthly precipitations vary between 0.0 $\mathrm{mm}$, for March, and $23.6 \mathrm{~mm}$, for June, being with $25.1 \mathrm{~mm}$ and $58.8 \mathrm{~mm}$, correspondingly, lesser than the multiannual monthly average value.

On the account of the above chart, we observe that the quantitative positive deviations are more numerous than the negative ones and act in accordance with the annual regime evolution of monthly mean multiannual.

The rain events generating hydrologic hazards are the torrential types supplied by summer months, short and swift, delivering a large volume of water in record time. Even the fall rainfalls with slightly reduced maximum can yield major destructive effects. 


\section{The pluviometric factor Angot}

The equation introduced by Angot can characterize any month of the year from the pluviometric standpoint without being conditioned or influenced by the number of days in a month. With the aid of this parameter it is possible to evaluate and determine the pluviometric features for each month across the year. If the value is subunitary (smaller than 1) the respective month displays a pluviometric deficit. If the obtained value, from calculus, equals 1 subsequently the month is regarded normal from the pluviometric factor point of view. Finally, if the value is overunitary (greater than 1) it results that the respective month produced a pluviometric surplus.

Amongst the most important applications of this parameter are: establishing the monthly pluviometric character, comparing precipitation amounts from different time frames and throughout the whole basin, evaluating the relief's susceptibility to erosion and landslides according to the pluviometric regime particularities and annual pluviometric characteristics for a catchment basin.

Regarding the drainage area of Câlnău the data interpolated was provided by weather station Buzău. This parameter was applied for monthly average amounts of precipitation recorded at this meteorology institution, within the 1980-2012 time frame. Table nr 1 displays that, within the Câlnău's catchment basin, in all 31 years of observations 8 months are generally rainy, whilst June and July are heavily abundant (over 2). Also, it is evidently that the draughtiest months are January, February, March and December, since the Angot factor value decreases under 1.

Table no. 1 - Monthly average value of pluviometric coefficient Angot at Buzău weather station, during the $1980-2012$ period.

\begin{tabular}{|c|c|c|c|c|c|c|c|c|c|c|c|c|}
\hline luna & I & II & III & IV & V & VI & VII & VIII & IX & X & XI & XII \\
\hline I.A. & 0.6 & 0.7 & 0.8 & 1.2 & 1.8 & 2.6 & 2.0 & 1.5 & 1.3 & 1.1 & 1.0 & 0.9 \\
\hline
\end{tabular}

The Angot coefficient, through its prime component allows the distinction from the range of values of data base employed from arid or wet time sections, from the array of analyzed data. According to acquired results can be determined the susceptibility classes of landscape to geomorphologic processes of interest, depending on fallen amounts of precipitation. For values of $\mathbf{K}$ over-unitary, the susceptibility rises directly proportional with its size, as depicted in Figure 9.

From the analysis of tables 2 and 3, below, in which are computed precipitation quantities on susceptibility classes according to Angot parameter, it is discovered:

- in the case of class I, which designates draughty months (January, February, March, October, November and December), fallen precipitations meet on average 
20 cases/month, respectively over $75 \%$ scarce amounts, presenting (according to Angot coefficient $K<\mathbf{0 . 9 9}$ ) a very small susceptibility of the land;

- susceptibility class II (small) is poorly represented assembling under 10 cases a month in all 31 years of precipitation measurements. Only April and June reach 10 in this time period. The probability of land erosion generation is under 15 $\%$ for the entire basin, and over $15 \%$ is registered in the two months mentioned above;

- the classes with medium susceptibility (III and IV) are the lowest occurring, these do not exceed 10 cases a month. The probability is small, generally under $15 \%$. Only for June in susceptibility class III the likelihood of development barely reaches 9 cases, while for the IV class, only May achieves 9 cases;

- the large susceptibility class $\mathrm{V}$ is depicted across the June interval, with a number of 15 cases in every month from all 31 years. More elevated quantitative values appear in May, July and August when it is overreached a rate of 5 cases per month.

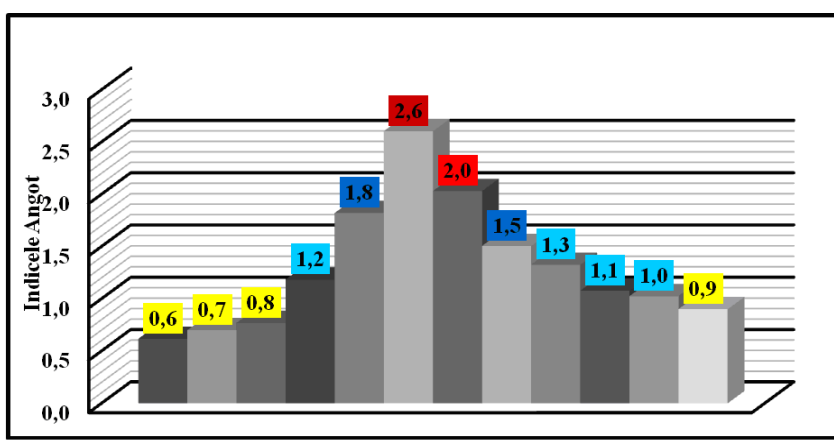

\begin{tabular}{|c|c|c|c|c|c|}
\hline Clase de susceptibilitate & f.mică & mică & medie & mare & f. mare \\
\hline valoare indice Angot & $<0.99$ & $1.00-1.49$ & $1.50-1.99$ & $2.00-2.49$ & $>2.50$ \\
\hline
\end{tabular}

Fig. 9 - Susceptibility classes of land for geomorphologic processes, according to precipitation quantities, after Angot parameter, for Buzău weather station, during the $1980-2012$ period.

Table no. 2 - The probability of geomorphologic processes occurrence (\%), according to precipitation quantities, on susceptibility classes, within the drainage basin of Câlnău

\begin{tabular}{|c|c|c|c|c|c|c|c|c|c|c|c|c|}
\hline class & ian & feb & mar & apr & may & iun & iul & aug & sept & oct & nov & dec \\
\hline I & 23 & 24 & 19 & 11 & 6 & 1 & 2 & 11 & 11 & 16 & 17 & 19 \\
\hline II & 6 & 2 & 7 & 10 & 4 & 0 & 10 & 8 & 8 & 3 & 5 & 6 \\
\hline III & 2 & 2 & 4 & 4 & 4 & 9 & 5 & 5 & 6 & 5 & 7 & 3 \\
\hline IV & 0 & 2 & 1 & 6 & 9 & 6 & 6 & 0 & 3 & 5 & 2 & 2 \\
\hline V & 0 & 1 & 0 & 0 & 8 & 15 & 8 & 7 & 3 & 2 & 0 & 1 \\
\hline
\end{tabular}


The occurrence probability of several extreme geomorphologic processes during the months of May - June - July- August, within the catchment area of Câlnău, exceeds $20-30 \%$.

Table no. 3 - Precipitation amounts on susceptibility classes according to Angot factor, at weather station Buzău, between 1980 -2012.

\begin{tabular}{|c|c|c|c|c|c|c|c|c|c|c|c|c|c|c|}
\hline $\begin{array}{c}\text { Year } \\
\text { /month }\end{array}$ & I & II & III & IV & $\mathbf{V}$ & VI & VII & VIII & IX & $\mathbf{X}$ & XI & XII & $\begin{array}{l}\text { sum } \\
\text { year }\end{array}$ & $\begin{array}{c}\text { mean } \\
\text { year }\end{array}$ \\
\hline 1980 & 1,6 & 0,1 & 1,5 & 1,8 & 4,9 & 1,5 & 3,5 & 1,6 & 1,2 & 0,7 & 1,5 & 2,3 & 22,1 & 1,9 \\
\hline 1981 & 0,9 & 0,9 & 1,9 & 1,9 & 2,7 & 1,6 & 1,0 & 1,6 & 0,8 & 1,5 & 2,2 & 1,9 & 18,9 & 1,6 \\
\hline 1982 & 0,1 & 0,5 & 0,3 & 2,1 & 0,7 & 1,9 & 2,4 & 1,9 & 0,1 & 0,4 & 0,6 & 1,3 & 12,3 & 1,0 \\
\hline 1983 & 0,1 & 0,7 & 0,3 & 0,7 & 1,3 & 4,8 & 2,0 & 3,9 & 0,2 & 0,7 & 1,1 & 0,3 & 16,0 & 1,3 \\
\hline 1984 & 1,4 & 2,1 & 1,8 & 2,1 & 2,1 & 3,5 & 3,0 & 0,8 & 0,8 & 0,3 & 1,9 & 0,7 & 20,4 & 1,7 \\
\hline 1985 & 1,5 & 0,9 & 0,2 & 0,5 & 2,0 & 5,9 & 1,7 & 1,1 & 0,3 & 0,2 & 1,6 & 0,3 & 16,1 & 1,3 \\
\hline 1986 & 0,9 & 2,6 & 0,5 & 0,4 & 0,3 & 1,7 & 1,7 & 0,4 & 0,4 & 1,0 & 0,3 & 0,6 & 10,8 & 0,9 \\
\hline 1987 & 0,5 & 1,0 & 0,3 & 1,0 & 2,1 & 2,1 & 1,3 & 0,8 & 0,5 & 0,7 & 2,8 & 0,8 & 13,9 & 1,2 \\
\hline 1988 & 0,8 & 2,1 & 2,0 & 1,8 & 2,0 & 3,6 & 1,2 & 0,4 & 2,4 & 0,9 & 0,5 & 0,8 & 18,4 & 1,5 \\
\hline 1989 & 0,1 & 0,4 & 0,4 & 1,0 & 0,9 & 4,6 & 0,5 & 1,7 & 1,7 & 0,9 & 0,9 & 0,2 & 13,3 & 1,1 \\
\hline 1990 & 0,1 & 0,9 & 0,0 & 2,2 & 2,6 & 2,1 & 1,2 & 0,6 & 1,1 & 1,2 & 0,1 & 2,7 & 14,7 & 1,2 \\
\hline 1991 & 0,2 & 0,9 & 0,3 & 1,4 & 5,6 & 3,9 & 5,3 & 1,8 & 0,6 & 1,7 & 1,3 & 0,5 & 23,5 & 2,0 \\
\hline 1992 & 0,0 & 0,4 & 1,1 & 0,7 & 2,0 & 3,9 & 2,2 & 0,9 & 0,3 & 1,3 & 0,2 & 0,5 & 13,5 & 1,1 \\
\hline 1993 & 0,0 & 0,6 & 1,3 & 0,7 & 2,4 & 2,0 & 2,2 & 0,9 & 1,0 & 0,2 & 1,9 & 0,7 & 13,8 & 1,2 \\
\hline 1994 & 0,4 & 0,2 & 0,4 & 0,7 & 0,9 & 2,4 & 2,9 & 1,1 & 1,4 & 2,3 & 0,1 & 1,2 & 13,9 & 1,2 \\
\hline 1995 & 1,0 & 0,1 & 0,6 & 0,8 & 1,9 & 3,3 & 1,4 & 1,3 & 4,2 & 0,3 & 1,8 & 0,9 & 17,4 & 1,4 \\
\hline 1996 & 1,4 & 0,8 & 0,9 & 1,4 & 3,2 & 1,7 & 1,5 & 1,0 & 2,2 & 0,4 & 2,1 & 1,8 & 18,4 & 1,5 \\
\hline 1997 & 0,3 & 0,1 & 1,1 & 2,4 & 2,4 & 2,8 & 4,7 & 3,0 & 0,6 & 2,2 & 2,3 & 2,1 & 23,8 & 2,0 \\
\hline 1998 & 1,6 & 0,3 & 0,9 & 1,0 & 1,5 & 1,9 & 0,2 & 1,1 & 1,5 & 2,3 & 1,0 & 0,4 & 13,6 & 1,1 \\
\hline 1999 & 0,7 & 0,2 & 0,8 & 2,0 & 1,1 & 5,0 & 1,0 & 3,2 & 4,2 & 2,2 & 0,3 & 1,3 & 22,1 & 1,8 \\
\hline 2000 & 0,6 & 1,2 & 0,6 & 1,1 & 0,6 & 2,2 & 1,4 & 0,6 & 2,0 & 0,1 & 0,4 & 0,4 & 11,3 & 0,9 \\
\hline 2001 & 0,4 & 0,9 & 0,6 & 1,4 & 1,2 & 2,3 & 1,9 & 0,3 & 3,7 & 0,3 & 0,3 & 0,4 & 13,6 & 1,2 \\
\hline 2002 & 0,5 & 0,1 & 0,4 & 0,9 & 0,6 & 2,5 & 5,2 & 1,3 & 1,3 & 3,0 & 1,2 & 1,0 & 18,0 & 1,5 \\
\hline 2003 & 1,2 & 0,5 & 0,4 & 1,0 & 0,4 & 0,8 & 3,0 & 1,1 & 1,6 & 2,5 & 0,6 & 0,4 & 13,5 & 1,1 \\
\hline 2004 & 1,1 & 0,6 & 1,0 & 0,5 & 1,4 & 1,8 & 2,1 & 3,8 & 1,9 & 0,6 & 1,1 & 0,6 & 16,3 & 1,4 \\
\hline 2005 & 0,5 & 1,6 & 1,0 & 1,0 & 3,0 & 3,6 & 5,6 & 4,0 & 1,8 & 0,2 & 1,6 & 1,0 & 24,7 & 2,1 \\
\hline 2006 & 1,3 & 0,2 & 1,1 & 2,2 & 2,6 & 3,7 & 2,2 & 3,1 & 0,5 & 0,5 & 0,2 & 0,5 & 17,9 & 1,5 \\
\hline 2007 & 0,6 & 0,3 & 1,5 & 0,6 & 1,5 & 1,5 & 1,3 & 4,4 & 1,3 & 2,3 & 1,5 & 1,0 & 17,7 & 1,5 \\
\hline 2008 & 0,1 & 0,1 & 0,3 & 1,9 & 2,3 & 1,8 & 1,1 & 0,3 & 1,2 & 1,7 & 1,1 & 0,9 & 12,6 & 1,1 \\
\hline 2009 & 0,1 & 0,4 & 1,4 & 0,9 & 1,7 & 3,0 & 1,1 & 1,2 & 1,1 & 1,7 & 0,9 & 0,9 & 14,3 & 1,3 \\
\hline 2010 & 0,8 & 1,9 & 0,9 & 1,2 & 2,1 & 2,7 & 1,5 & 0,7 & 1,6 & 1,5 & 0,4 & 1,6 & 17,0 & 1,4 \\
\hline mean & 0,6 & 0,7 & 0,8 & 1,2 & 1,8 & 2,6 & 2,0 & 1,5 & 1,3 & 1,1 & 1,0 & 0,9 & 15,6 & \\
\hline
\end{tabular}

\section{Conclusions}

Precipitations have a direct impact on the landscape through pluvial denudation, installation of channels and trenches, ditches apparition, gorges and torrential organisms. Furthermore, as a major and direct impact, we are reminding the pluvial factor as an active part for the onset of landslides. 
Even more, precipitations have an indirect impact on relief erosion through the rise and fall of liquid and solid debits of rivers within a catchment basin, rivers playing an important role in permanent erosion of the land.

The turbidity is determined by the presence of colloidal suspensions and gatherings of organic and inorganic nature, which rise quantitatively in the direction upstream - downstream. They register great abundances in the spring and after the downpours in summer.

Regarding the drainage area of Câlnău, the average specific discharge of alluviums in suspension is of $54 \mathrm{t} / \mathrm{ha} / \mathrm{an}$, the largest multiannual mean in the country. Câlnău's river basin is included on the map of average discharge specific (after I.M.H., 1988) to the most afflicted zones by erosion.

As a result of the mentioned processes an intense accumulation of sediments take place near the mouth of confluent rivers and torrential organisms allowing the formation large alluvial fans. These microstructures have resulted due to exerted intense erosion, especially in the summer months, according to Angot parameter, which measures the land's susceptibility to erosion wielded by atmospheric precipitations. During the more arid months or with scarce precipitations the terrain is prepared to be eroded, through the drying of sediment deposits from banks and versants which do not present a band of protective forests.

\section{References}

Băloiu V. (1980), The improvement of drainage basins and water courses, Ed. Ceres, Bucharest.

Cioacă A., Dinu Mihaela (1999), Model of geomorphologic cartography of processes on versant from Câlnău Valley, Sub-Carpathians Vrancei zone, Geographic Comunications III, Ed. University from Bucharest.

Dragotă Carmen - Sofia (2006), Exceeding precipitations in Romania, Ed. Romanian Academy, Bucharest.

Ielinicz M., Pătru Ileana, Ghincea Mioara (2003), Romanian Sub-Carpathians, Ed. University, Bucharest.

Minea I.S. (2011), Rivers of Buzău basin- hydrographical and hydrological considerations, Ed. Alpha MDN, Buzău.

Pișota I., Zaharia Liliana (2001, 2002), Hydrology, Ed. University from Bucharest.

*** (1971), Buzău County - counties of homeland, Ed. Romanian Academy, Bucharest.

*** (1992), Romanian Geography, vol. IV, Ed. Romanian Academy, Bucharest.

$* * *$ (2008), Romanian Clime, ANM, Ed. Romanian Academy, Bucharest

*** Topographic Maps, scale 1: 50000.

www.inhga.ro 